\title{
Split-hand/foot malformation - molecular cause and implications in genetic counseling
}

\author{
Anna Sowińska-Seidler • Magdalena Socha • \\ Aleksander Jamsheer
}

Received: 29 July 2013 /Revised: 21 September 2013 / Accepted: 23 September 2013 / Published online: 27 October 2013

(C) The Author(s) 2013. This article is published with open access at Springerlink.com

\begin{abstract}
Split-hand/foot malformation (SHFM) is a congenital limb defect affecting predominantly the central rays of the autopod and occurs either as an isolated trait or part of a multiple congenital anomaly syndrome. SHFM is usually sporadic, familial forms are uncommon. The condition is clinically and genetically heterogeneous and shows mostly autosomal dominant inheritance with variable expressivity and reduced penetrance. To date, seven chromosomal loci associated with isolated SHFM have been described, i.e., SHFM1 to 6 and SHFM/SHFLD. The autosomal dominant mode of inheritance is typical for SHFM1, SHFM3, SHFM4, SHFM5. Autosomal recessive and X-linked inheritance is very uncommon and have been noted only in a few families. Most of the known SHFM loci are associated with chromosomal rearrangements that involve small deletions or duplications of the human genome. In addition, three genes, i.e., TP63, WNT10B, and DLX5 are known to carry point mutations in patients affected by SHFM. In this review, we focus on the known molecular basis of isolated SHFM. We provide clinical and molecular information about each type of abnormality as well as discuss the underlying pathways and mechanism that contribute to their development. Recent progress in the understanding of SHFM pathogenesis currently allows for the identification of causative genetic changes in about $50 \%$ of the patients affected by this condition. Therefore, we propose a diagnostic flow-chart helpful in the planning of molecular genetic tests aimed at identifying disease causing
\end{abstract}

\footnotetext{
A. Sowińska-Seidler $(\bowtie) \cdot$ M. Socha $\cdot$ A. Jamsheer Department of Medical Genetics, Poznan University of Medical Sciences, 55 Grunwaldzka Street, Pav. 15, 60-352 Poznan, Poland e-mail: asowinskaseidler@gmail.com

A. Jamsheer $(\square)$

NZOZ Center for Medical Genetics GENESIS, Poznan, Poland e-mail: jamsheer@wp.pl
}

mutation. Finally, we address the issue of genetic counseling, which can be extremely difficult and challenging especially in sporadic SHFM cases.

Keywords DLX5 $\cdot$ SHFM $\cdot$ SHFLD $\cdot$ Split-hand/foot malformation $\cdot$ TP63 $\cdot$ WNT10B

\section{Introduction}

Split-hand foot malformation (SHFM) also known as ectrodactyly is a congenital limb defect affecting predominantly the central rays of hands and/or feet. It may manifest either as an isolated trait or part of a multiple congenital anomaly syndrome. SHFM occurs with the incidence of about 1 per 18,000 liveborn infants and accounts for $8-17 \%$ of all limb malformations (Czeizel et al. 1993). SHFM is a clinically heterogeneous abnormality, which ranges from a relatively mild defect, such as hypoplasia of a single phalanx or syndactyly, to the aplasia of one or more central digits (i.e., classical cleft also known as lobster-claw anomaly, see Fig. 1a). In its most severe form - monodactyly - the defect is characterized by the aplasia of both central and preaxial rays of the autopod (Fig. 1b) (Jamsheer 2008). As a consequence, interindividual and intrafamilial variability of the SHFM is very high. Furthermore, variable expressivity of this feature can be so significant, that a different pattern of anomaly is seen in each limb of the same individual patient (Temtamy and McKusick 1978). The condition is mostly sporadic, familial forms are uncommon. In the majority of cases SHFM undergoes autosomal dominant mode of inheritance with reduced penetrance but other types of inheritance have also been reported (see Table 1).

In this review, we focus on the known molecular basis of isolated SHFM. We provide clinical and molecular information about each type of abnormality as well as discuss the 


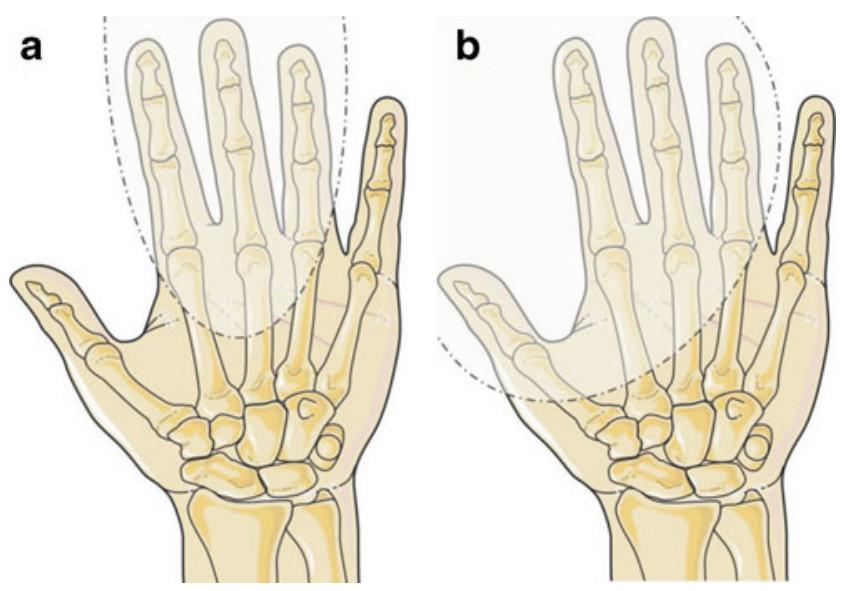

Fig. 1 Schematic presentation of the different anatomical types of SHFM (a) classical cleft characterized by the aplasia of the central rays of the autopod; (b ) monodactyly characterized by the aplasia of both central and preaxial rays of the autopod

underlying pathways and mechanism that contribute to their development (see Table 1). Recent progress in the understanding of SHFM pathogenesis currently allows for identification of causative genetic changes in about $50 \%$ of the patients affected by this condition. Therefore, we propose a diagnostic flow-chart helpful in the planning of molecular genetic tests aimed at identifying disease causing mutation. Finally, we address the issue of genetic counseling, which can be extremely difficult and challenging especially in sporadic SHFM cases.

\section{Developmental aspects of SHFM formation}

The developing limb bud consists of two cell layers: highly proliferating mesenchymal cells covered by the ectodermal cells. The formation of limb bud is mediated by signaling molecules produced by three specialized cell groups - the apical ectodermal ridge (AER), the progress zone (PZ), and the zone of polarizing activity (ZPA). The interaction between these three regions determine the patterning of the limb in three spatial dimensions: proximo-distal, antero-posterior, and dorso-ventral. A number of signaling molecules and transcription factors such as fibroblast growth factors (FGFs), bone morphogenic proteins (BMPs) or WNT and MSX proteins produced by AER keep the neighboring mesenchymal cells in constant proliferation and undifferentiated state, giving rise to PZ (Gurrieri et al. 2002). Constantly proliferating cells of $\mathrm{PZ}$ determine the proximo-distal polarity of the limb bud. Failure in maintaining the AER affects the formation of the autopod and leads to the development of SHFM phenotype. Not only genetic, but also environmental factors that include apoptosis in the AER can cause SHFM (Sulik and Dehart 1988). Several genetic factors responsible for SHFM development have been identified to date.

\section{Known SHFM loci}

SHFM1 (7q21)

Split hand/foot malformation type 1 (SHFM1) is caused by chromosomal rearrangements of the 7q21.3-q22.1 region (Fig. 2a). They occur mostly as de novo mutational events, but can also be inherited in an autosomal dominant manner, with reduced penetrance and variable expression. Genomic alteration of the SHFM1 locus can be either associated with isolated or syndromic limb malformation. For instance, sensorineural deafness is noted in $35 \%$ of patients, while EEC symptoms (ectrodactyly-ectodermal dysplasia-cleft lip/palate) are much less frequent (Tackels-Horne et al. 2001). Aberrations within this region involve translocations, inversions, duplications, and most frequently, deletions. The minimal overlapping region for SHFM1 encompass several genes involved in limb development, such as DSS1, DLX5, and DLX6 (Crackower et al. 1996; Duijf et al. 2003). Crackower et al. (1996) found that the murine ortholog of DSS1 is predominantly expressed in the mesenchyme of the developing limb bud, facial primordia, as well as in the branchial arches, genital tubercle, and dermis. It was proposed that the reduced expression of DSS1 during the early stages of embryogenesis might be responsible for SHFM (Crackower et al. 1996). However, the two other genes located within the 7q21-22 critical region, i.e., $D L X 5$ and $D L X 6$, were found to be more attractive candidate genes for SHFM. Both belong to the members of the Wnt signaling pathway, which is important in limb skeleton development and morphogenesis (van Silfhout et al. 2009). Expression of the murine orthologs - Dlx 5 and Dlx6 - has been detected in the AER of the embryonic limb buds, in the pharyngeal arches, in the osteoblasts of developing bones, and in the inter neurons of the basal forebrain (Simeone et al. 1994; Acampora et al. 1999; Lo Iacono et al. 2008). Both gene products are critical for the maintenance of the proliferation of medial AER cell population in the developing limb bud (Robledo et al. 2002). The role of $D L X 5 / D L X 6$ in limb morphogenesis has been demonstrated in mouse models, where the double knock-out of both genes $\left(D l \times 5 / D l x 6^{-1}\right)$ resulted in a typical ectrodactyly as well as craniofacial and inner ear abnormalities. Interestingly, the deletion of neither gene alone has led to ectrodactyly or other limb phenotype (Robledo et al. 2002). Of note, the SHFM in Dlx5/ $D l x 6^{-/-}$null mice could be rescued by solely overexpressing Dlx 5 in the AER suggesting Dlx5 and Dlx6 redundancy in limb development (Robledo et al. 2002). On the other hand, overexpression of $D l x 5$ in wild-type mice has no negative effect on limb formation. The hypothesized $D L X 5$ and/or $D L X 6$ biological activity is that they function as repressors of downstream target genes, which in the absence of both $D L X 5$ and DLX6 are overexpressed, thereby giving rise to the reduction of cell proliferation within the AER (Robledo et al. 2002). 


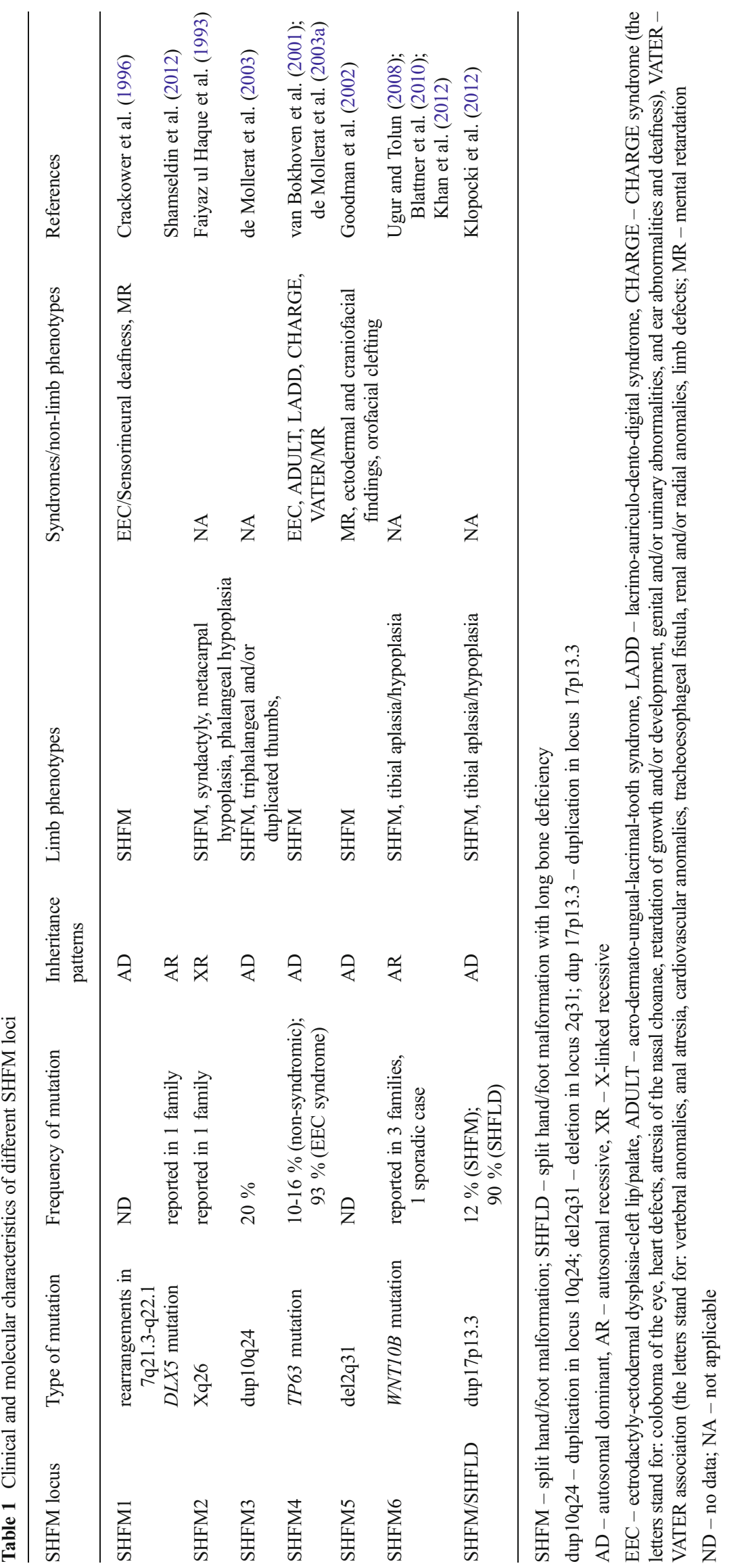




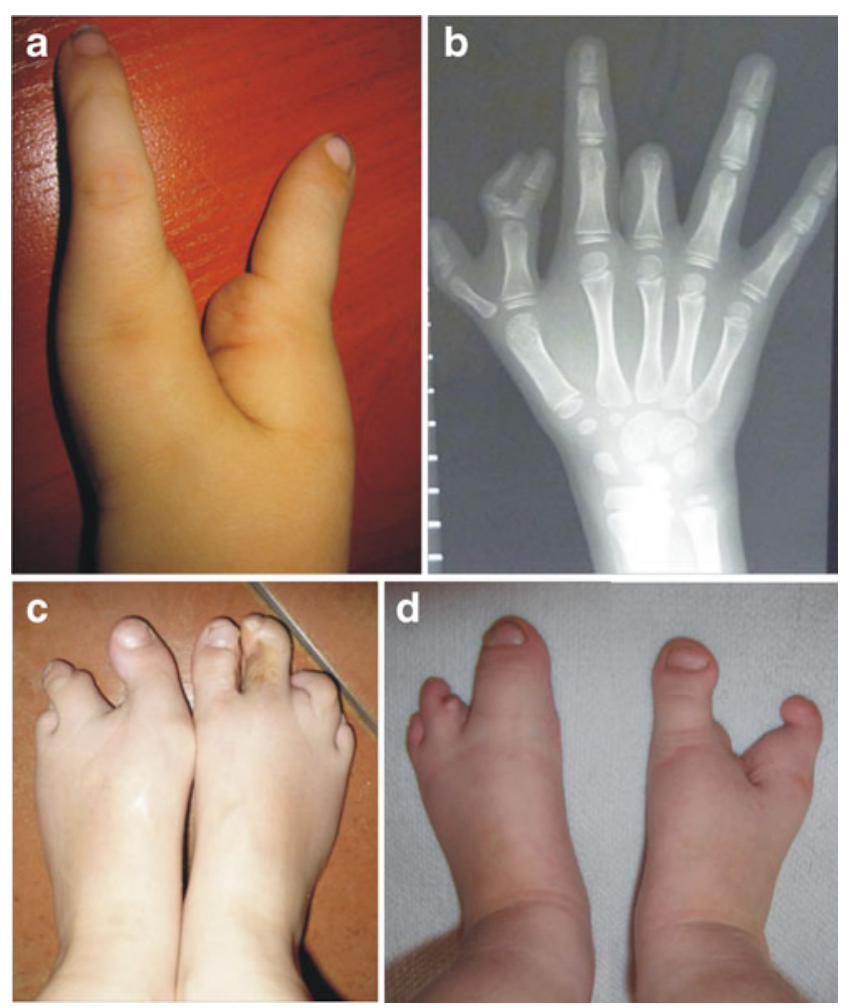

Fig. 2 Limbs of the SHFM patients with different molecular origin of the defect (a) male proband with a classical cleft of the hands carrying a balanced chromosomal translocation $\mathrm{t}(7 ; 12)(\mathrm{q} 21.2 ; \mathrm{q} 21.3)$ involving SHFM1 locus; $(\mathbf{b}, \mathbf{c})$ female proband carrying a typical 10q24 duplication of the SHFM3 locus showing ectrodactyly and preaxial polydactyly of the hands as well as ectrodactyly of the feet; (d) female proband with a classical cleft of the feet carrying a nonsense heterozygous point mutation c.G1974A(p.W658X) in the TP63 gene

The mouse model for SHFM1 varies significantly from the clinical setting observed in human individuals. While mouse double $D l x 5 / D l x 6^{-/}$knock-out is recessive, human patients develop the condition if the aberration affects only a single copy of SHFM1 locus. Consequently, human SHFM1 deletions are inherited in an autosomal dominant manner. Thus, based on the interspecies differences, one can hypothesize that human limb development is more sensitive to reduced levels of DLX5/6 proteins (Robledo et al. 2002). To make things more complex, haploinsufficiency of SHFM1 locus is not the only pathomechanism leading to limb defect in human individuals. Alternative mechanisms occur in the case of chromosomal translocations or inversions affecting SHFM1 locus and include "position effect" on $D L X 5 / D L X 6$ genes resulting either from the disruption of the regulatory elements or from changing the regulatory landscape on 7q21 (Merlo et al. 2002). Transcriptional regulation of $D L X 5$ and $D L X 6$ genes has been investigated by several authors. Lo Iacono et al. (2008) have shown that DLX proteins and p63 transcription factor, encoded by TP63 gene, colocalize in the AER and that p63 acts as an upstream regulator of Dlx 5 and Dlx6 transcription via binding to their cis-acting regulatory elements at the promoter level. Heterozygous mutations in TP63 give rise to limb malformations in multiple congenital syndromes as well as to isolated SHFM (SHFM4 - described below). Genomewide profiling of TP63 cis-regulatory elements and target genes, reported by Kouwenhoven et al. identified $\mathrm{p} 63$ binding site - SHFM1-BS1, that can function as an enhancer element controlling the expression of $D L X 6$, and possibly $D L X 5$, by physical interaction with $D l x 6$ promoter (zebrafish and mice assay) (Kouwenhoven et al. 2010). The expression of $D l \times 5 /$ Dlx6 is reduced upon p63 inactivation (Lo Iacono et al. 2008) or disruption of regulatory elements controlled by $\mathrm{p} 63$ (Kouwenhoven et al. 2010). Recent studies in the transcriptional regulation of $D L X 5 / 6$ resulted in the identification of additional enhancers located in protein coding sequences of the neighboring genes. Birnbaum et al. (2012) have described two novel limb exonic enhancers, (eExons) DYNC1I1 eExon 15 and 17, that reside proximal to $D L X 5 / 6$. With the use of mouse and zebrafish transgenic enhancer assays the authors characterized the enhancer activity of DYNC1I1 eExon 15 in the AER and DYNC1I1 eExon 17 in the anterior mesenchyme, and thus showed their regulatory influence on $D l \times 5 / 6$ limb expression (Birnbaum et al. 2012). The above findings gave a new insight to putative genes, regulatory elements and novel disease mechanisms in the etiopathogenesis of SHFM1.

Recently, Shamseldin et al. (2012) studied a consanguineous family of putative autosomal recessive SHFM and found that homozygous DLX5 missense mutation located in the homeodomain was causative for the limb phenotype. Thus, the authors provided the first strong evidence on direct $D L X 5$ involvement in the pathogenesis of human SHFM1 (Shamseldin et al. 2012).

\section{SHFM2 (Xq26)}

To date only one family has been reported with X-lined inheritance of SHFM (Ahmad et al. 1987). The authors described a consanguineous Pakistani family of which 36 individuals manifested full-blown SHFM (33 males and three females). The patients exhibited monodactylous or bidactylous hands and typical, but slightly variable feet. About half of the heterozygous females showed mild hand and/or foot malformations. Cytogenetic studies excluded the possibility of $\mathrm{X} /$ autosome translocations (Ahmad et al. 1987). The X-linked inheritance of the trait in this family was later confirmed by linkage analysis which mapped the SHFM2 locus to Xq26 (Faiyaz ul Haque et al. 1993). Two genes located in this region, FGF13 and TONDU, may potentially be the candidate genes for SHFM2, however no coding mutations have been found in the above genes in patients with SHFM (Vaudin et al. 1999). 


\section{SHFM3 (10q24)}

The locus for SHFM3 maps to chromosome 10q24 and the causative rearrangement involves a submicroscopic tandem duplication spanning a region of $325-570 \mathrm{~kb}$ (de Mollerat et al. 2003; Dimitrov et al. 2010). Duplications of the SHFM3 locus are probably the most frequent cause of SHFM in humans and accounts for about $20 \%$ of cases (Klopocki et al. 2012). Several genes potentially contributing to the SHFM phenotype located within the duplicated region include DACTYLIN (SFHM3), BTRC, POLL, FGF8, and LBXI. The SHFM3 duplication is inherited in an autosomal dominant manner and associates mostly with the non-syndromic classical SHFM, which is frequently accompanied with preaxial abnormalities such as triphalangeal and/or duplicated thumbs (see Fig. 2b and c) (Everman et al. 2006).

The naturally occurring Dactylaplasia (Dac) mouse is considered to be an animal model for human SHFM3. Both human and mouse SHFM3 regions (Dac locus maps to chromosome 19) share a high degree of homology. The Dac mice display the absence of central digits, hypoplasia or aplasia of metacarpal/ metatarsal bones and syndactyly. The phenotype is inherited in a semi-dominant manner, i.e., heterozygotes present with the absence of central digits of hind- and forelimbs, whereas homozygotes show monodactyly (Johnson et al. 1995; Sidow et al. 1999). The mechanism that underlies the loss of digits in Dac mutants involves increased cell death in a specific portion of the AER. In Dac/+ (heterozygous) mice only the central part of AER is degenerated, whereas in Dac/Dac (homozygous) mice both the central and the anterior part of the AER are affected (Seto et al. 1997; Crackower et al. 1998).

The mechanism by which the duplication of the SHFM3 critical region gives rise to the limb defect remains unknown. Possible explanations are that either the aberrant DACTYLIN transcript exhibits a dominant negative effect on wild-type alleles or that the overexpression of other duplicated genes takes place, among which BTRC ( $\beta-T R C P)$ is of particular interest (de Mollerat et al. 2003). BTRC functions as a ubiquitination factor of proteins involved in several signaling transduction pathways such as $\mathrm{Wnt} / \beta$-catenin, $\mathrm{SHH}$, and NF-KB (Maniatis 1999). All these pathways are involved in limb development, therefore dysregulation of BTRC expression in a dosage dependent manner may be responsible for the SHFM3 phenotype (de Mollerat et al. 2003). FGF8 also located within the SHFM3 critical region induces and regulates the limb bud patterning via AER signaling. Fgf 8 mouse ortholog is highly expressed throughout the AER. Its inactivation in early limb ectoderm causes hypoplasia/aplasia of specific distal skeletal elements and alters the expression of Shh and Bmp2 genes (Lewandoski et al. 2000; Moon and Capecchi 2000). To date, no pathogenic mutations have been reported in any of the putative candidate genes. Another mechanism that should be taken into consideration is a cis- acting position effect that gives rise to the skewed expression of genes flanking the breakpoints of the duplication (de Mollerat et al. 2003).

\section{SHFM4 (3q27; TP63)}

A fourth SHFM locus was mapped to $3 \mathrm{q} 27$ region and is directly associated with mutations in TP63 (also known as P63) gene (Ianakiev et al. 2000). This is the only locus of monogenic etiology inherited in an autosomal dominant fashion. The protein encoded by TP63 gene is a transcription factor homologous to tumor suppressor gene TP53. TP63 plays a crucial role in the embryonic development since it regulates the formation and differentiation of the AER. It functions as a key regulator of ectodermal development by maintaining the proliferative potential of ectodermal cells (Mills et al. 1999; Yang et al. 1999). TP63 controls the epithelial stratification process via regulation of AERrestricted genes (Koster et al. 2004). Mice lacking p63 fail to form normal ectodermal structures, which is manifested as developmental abnormalities of the skin, hair, teeth, craniofacial skeleton, mammary glands, and limbs, including ectrodactyly or other partial limb truncations or amelia (Mills et al. 1999; Yang et al. 1999). The lack of morphologically recognizable AER in the limb buds of $p 63$-null mice results in amelia and suggests that p63 is essential for the initiation of this structure (Ianakiev et al. 2000).

TP63 mutations in humans are responsible for approximately $10-16 \%$ of isolated SHFM cases (see Fig. 2 d). On the other hand, mutations in this gene are found in $93 \%$ of patients affected by EEC syndrome in which limb malformations occur as a part of the phenotypic spectrum (van Bokhoven et al. 2001; de Mollerat et al. 2003a).

\section{SHFM5 (2q31)}

Deletions of chromosome $2 \mathrm{q} 31$ region encompassing the entire HOXD gene cluster (HOXD1-HOXD13) has been described in patients with SHFM, including monodactyly (Ramer et al. 1990; Boles et al. 1995; Del Campo et al. 1999). However, Goodman et al. have suggested that a locus for SHFM5 is associated with the deletions in $2 \mathrm{q} 31$ region encompassing the $5-\mathrm{Mb}$ interval centromeric to EVX2 gene, which is located upstream of the HOXD cluster (Goodman et al. 2002). The authors reported two families with limb malformations. In the first family, father and daughter were affected with synpolydactyly (SPD) caused by a deletion that involved 5' part of the HOXD gene cluster (HOXD9 HOXD13) and extended only $85 \mathrm{~kb}$ upstream of HOXD13 encompassing $E V X 2$ gene. In the second family, the proband presented with bilateral split foot and deletion at 2q31-q33 comprising the entire HOXD cluster (HOXD1-HOXD13), $E V X 2, D L X 1$ and $D L X 2$ up to microsatellite marker 
D2S294, located approximately $5 \mathrm{Mb}$ centromeric to $E V X 2$ (Goodman et al. 2002). On the 3 ' side of the cluster, the four remaining HOXD genes (HOXD1, HOXD3, HOXD4, $H O X D 8)$ are not expressed in the developing limbs (Goodman et al. 2002). Taken together, these findings indicated that haploinsufficiency of 5' $H O X D$ cluster causes SPD, but not SHFM, which is related to a locus located centromeric to EVX2 on $2 \mathrm{q} 31$. Two genes $D L X 1$ and $D L X 2$, that lie within this locus are putative candidate genes for ectrodactyly as both are expressed in the AER and PZ (Goodman et al. 2002). However, neither heterozygous, nor homozygous $D l x 1, D l x 2$, and double $D l x 1 /$ Dlx2 knock-out mice have limb malformations (Qiu et al. 1997). Furthermore, no pathogenic mutations in those genes have been identified so far, thus their role in the pathogenesis of SHFM remains elusive.

\section{SHFM6 (12q13; WNT10B)}

To date only three consanguineous families and one sporadic case exhibiting autosomal recessive inheritance of SHFM have been reported (Ugur and Tolun 2008; Blattner et al. 2010; Khan et al. 2012; Shamseldin et al. 2012). As mentioned above, in one family SHFM was caused by a homozygous $D L X 5$ point mutation in SHFM1 locus (Shamseldin et al. 2012). The three remaining cases of autosomal recessive SHFM resulted from homozygous mutations in WNT10B, a gene located within the SHFM6 locus. WNT proteins act as ligands in a variety of signaling pathways and play a major role in limb morphogenesis and development (Yang 2003). Disease causing mutation in this gene was first described by Ugur and Tolun (2008), who identified a homozygous missense variant (p.R332W) in all affected members of the Turkish consanguineous family (with the exception of one mildly affected individual). Importantly, the homozygous mutation was also identified in one unaffected individual from the same family, which led to the assumption that the reported $W N T 10 B$ variant is necessary, but not sufficient to produce SHFM. Therefore, the authors hypothesized the coexistence of a second locus that contributes to the phenotype or the presence of a suppressor mutation in the unaffected individual (Ugur and Tolun 2008). Another description of an autosomal recessive SHFM concerned a Swiss patient with a homozygous 4-bp duplication in WNT1OB. Interestingly, this was the sporadic case that gave a new insight to the genetic counseling of all patients with sporadic SHFM, as they were generally considered to have dominantly inherited de novo mutations (Blattner et al. 2010). The recently reported novel homozygous mutation in WNT1OB (p.T329R) was detected in a consanguineous Pakistani family with autosomal recessive SHFM in all of the affected family members (Khan et al. 2012).

\section{Other SHFM loci}

Split hand/foot malformation with long bone deficiency (SHFLD) involving tibia and fibula is most commonly associated with the duplication of $17 \mathrm{p} 13.3$ locus. The first report by Lezirovitz et al. (2008) of a large Brazilian family mapped the SHFLD to an $841 \mathrm{~kb}$ interval at $17 \mathrm{p} 13.1-17 \mathrm{p} 13.3$ (Lezirovitz et al. 2008). Next, Klopocki et al. (2012) found that the defect is caused by a tandem duplication and narrowed down the region to the smallest region of overlap, including only the single BHLHA9 gene. The authors have investigated the expression pattern of BHLHA9 mouse and zebrafish orthologs at different embryonic stages. The results of this study indicated that Bhlha9 expression in both species was restricted to the AER of the limb bud mesenchyme. To further investigate the role of BHLHA9 in limb development the authors have performed knockdown experiments in zebrafish embryos, which revealed that loss of protein function resulted in shortening of the pectoral fins, thus confirming its role in limb development (Klopocki et al. 2012). Duplication in the 17 p 13.3 locus is responsible for approximately $12 \%$ of SHFM without long bone involvement. One of the most interesting clinical aspects of this study was that SHFLD related to $17 \mathrm{p} 13.3$ locus showed a high degree of nonpenetrance with sex bias, as approximately $50 \%$ of the duplication carriers (particularly females) were unaffected. This may be due to the coexistence of another factor related to a hormone responsive element or modifier on the Xchromosome that together with $17 \mathrm{p} 13.3$ duplication led to the development of SHFLD (Klopocki et al. 2012).

To date, there has been a single report describing a male patient who manifested SHFM probably due to a de novo $1 \mathrm{Mb}$ deletion in chromosome 19p13.11. One gene included in this duplication - EPS15L1 - was suggested by the authors as a candidate gene for SHFM (Aten et al. 2008). EPS15L1 protein acts as a substrate for the tyrosine kinase activity of the epidermal growth factor receptor (EGFR), which has been associated with limb development (Wong et al. 1995). An indirect evidence for the role of EPS15L1 in SHFM was provided by Bens et al. (2011) who described a patient with a de novo deletion partly overlapping with the copy number variant (CNV) detected by Aten et al. (2008). Interestingly, both patients shared most of the phenotypic features except from SHFM and also EPS15L1 gene remained non-deleted in a patient without limb phenotype (Bens et al. 2011). This genotype-phenotype correlation substantiates the hypothesis on EPS15L1 as a potential candidate gene for SHFM, which nevertheless needs further support.

Frequent SHFM syndromes

As mentioned above, ectrodactyly may occur either as an isolated malformation or coexists with other congenital 
anomalies such as cleft lip/palate, ectodermal dysplasia or developmental delay/mental retardation. There are several relatively frequent syndromes in which ectrodactyly occurs as one of the multiple congenital anomalies. The most common involve EEC syndrome (ectrodactyly-ectodermal dysplasia-cleft lip/palate syndrome), EEC syndrome without cleft lip/palate, LADD syndrome (lacrimo-auriculo-dentodigital syndrome), ADULT syndrome (acro-dermato-unguallacrimal-tooth syndrome), CHARGE syndrome or VATER association (see Table 1).

\section{Diagnostic aspects and genetic counseling}

Diagnosis in a patient presenting with SHFM should be achieved on the basis of both careful clinical examination as well as relevant cytogenetic and/or molecular tests. Clinical features and the underlying genetic defects of SHFM have been described in detail in the above paragraph, hence we focus here on the diagnostic approach and genetic counseling.

As described above, at least seven SHFM types (i.e., six basic from SHFM1 to SHFM6 and one additional SHFM/ SHFLD) have been distinguished in the literature (Blattner et al. 2010; Klopocki et al. 2012). The most common mode of inheritance for split hand/foot malformation is an autosomal dominant one with reduced penetrance (Duijf et al. 2003), although autosomal recessive (Elliott et al. 2005; Blattner et al. 2010; Shamseldin et al. 2012), as well as X-linked inheritance (Ahmad et al. 1987) have also been reported. Sporadic cases with isolated SHFM most likely result from de novo mutations. In such a setting the recurrence risk for the proband's sib is very low, while for the proband's offspring is high and reaches $30-50 \%$ (Blattner et al. 2010). Considering reduced penetrance, variable expression or non-Mendelian inheritance as well as segregation distortion and sex bias (Klopocki et al. 2012) with overtransmission of the genetic alteration from affected fathers to sons (Jarvik et al. 1994), it becomes clear, that genetic counseling in SHFM cases is rather difficult and challenging, not only in sporadic but also in familial cases. Furthermore, many of the SHFM cases seem to originate from complex set of mutations/chromosomal aberrations and have to be regarded as two- or multigenic disorders. Consequently, if genetic counseling in SHFM is to be reliable and informative it should be based on the panel of relevant genetic testing. Importantly, for the significant proportion of the SHFM patients who are tested for all known causative alterations, genetic abnormalities remain uncovered. Hence, for those patients the exact recurrence risk is unknown and has to be predicted based on empirical data.

The first tier genetic test should account for the frequency of the underlying causative SHFM changes. The largest cohort of SHFM patients described to date comprised 56 cases (Klopocki et al. 2012). The most frequent genetic abnormalities in this group were submicroscopic duplications of 10q24.3 (SHFM3) and $17 \mathrm{p} 13.3$ (SHFM/SHFLD) responsible for $20 \%$ and $16 \%$ of cases, respectively. Based on this finding and on our own diagnostic experience we propose that SHFM testing should begin with copy number screening for 10q24 followed by 17 p13.3 loci. Array CGH (aCGH) would be the method of choice as it allows not only for the detection of these changes, but also for the identification of other unbalanced chromosomal aberrations such as submicroscopic rearrangements affecting different loci (i.e., SHFM1 or SHFM5). Since the vast majority of SHFM related aberrations involve around several hundred $\mathrm{kbs}$ of genomic DNA, the resolution of aCGH platforms does not have to be particularly high. Routine diagnostic arrays with the resolution of about $180 \mathrm{k}$ oligonucleotide probes per haploid genome are sufficient for identifying most of the underlying CNVs. Alternatively, aCGH can be substituted by quantitative assays (such as MLPA or qPCR) for all known genomic SHFM loci. To our knowledge, there are no commercially available validated diagnostic MLPA or qPCR kits, therefore such tests have to be developed in-house and as a consequence they are less reliable. In some SHFM cases the conventional karyotyping is sufficient for diagnosis, as it can reveal a large chromosomal aberrations, such as deletions or translocations involving the 7q21-q22 region (Crackower et al. 1996; Duijf et al. 2003; Elliot and Evans 2006), namely SHFM1 locus (Elliott et al. 2005) or deletions of 2q31 (SHFM5) (Goodman et al. 2002; Elliott et al. 2005). Thus, basic GTG banding is still useful next to molecular testing, as it is a relatively cheap and informative assay in a subset of patients linked to SHFM1 or SHFM5 loci. An important advantage of karyotyping in reference to aCGH is its ability to detect balanced chromosomal rearrangements, for instance translocations, which are not infrequent in SHFM1 locus. Other patients presenting with isolated SHFM described so far (except for the tiny proportion of SHFM3) carry normal karyotypes. Conventional GTG banding should always be considered in syndromic SHFM especially in the case of association with additional congenital anomalies or mental retardation which are most common and severe in SHFM5 cases (Elliot and Evans 2006). Another important diagnostic test in SHFM patients is the TP63 gene sequencing. This is because point mutations in this gene give rise to about $10-16 \%$ of isolated SHFM and may occur either de novo or have autosomal dominant inheritance with a $50 \%$ recurrence risk. The TP63 mutations show rather complete penetrance but highly variable expressivity (de Mollerat et al. 2003a).

The recently discovered SHFM6 locus contains the $W N T 10 B$ gene (Ugur and Tolun 2008). SHFM6-dependent defect is inherited in an autosomal recessive manner and is caused by either homozygous or compound heterozygous $W N T 10 B$ mutations. Other autosomal recessive changes resulting in SHFM were very recently associated in a single family with homozygous $D L X 5$ mutations in the homeodomain of the gene (Shamseldin et al. 2012). In the recessive inheritance model, the recurrence risk for proband's sib is $25 \%$ 
Fig. 3 Diagnostic flow-chart useful in the planning of SHFM genetic diagnostics. Order of tests account for the relative frequencies of different SHFM causes as well as prices (in the case of karyotyping vs TP63 sequencing). $\mathrm{CNV}$ - copy number variation, $\mathrm{AD}$ autosomal dominant, AR autosomal recessive, WES whole exome sequencing, WGS whole genome sequencing
Table 2 Sequences of the primers used for TP63, WNT10B and $D L X 5$ genes amplification and sequencing

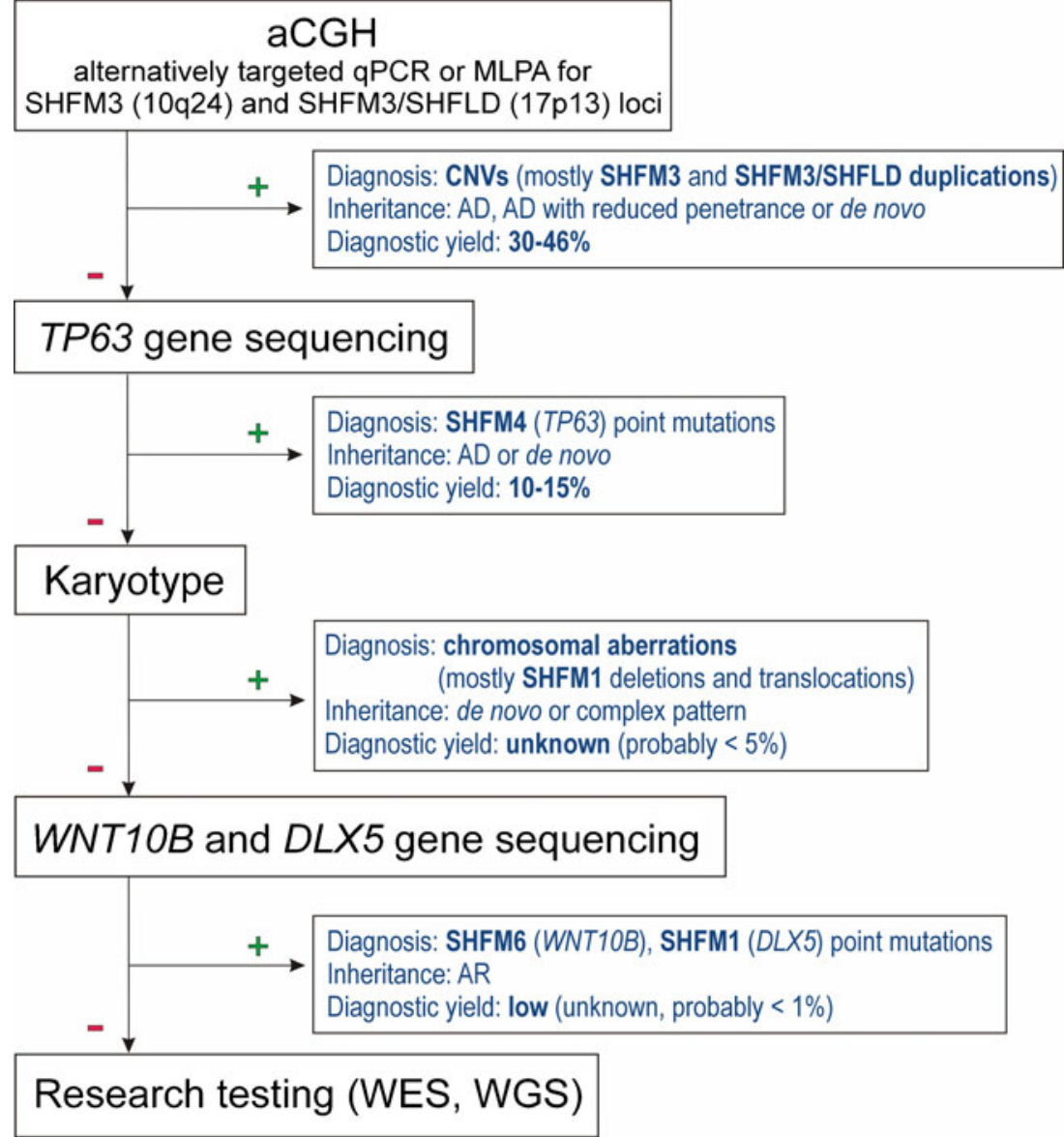

\begin{tabular}{lll}
\hline Exon name & F primer sequence 5'-3' & R primer sequence 5'-3' \\
\hline TP63_e1 & TCCCGGCTTTATATCTATATATAC & GACACATTCATAATACACAAGGCAC \\
TP63_e2 & TCCACTTGGGTTTTCATGATAGAG & GTAAGCAATATTTTGACCACCCAC \\
TP63_e3 & GCTTGTTGTTAACAACAGCATG & GAAAAGACAGGTTTAACAGAGC \\
TP63_e4 & GTGAAGTGCTTCCGACGTG & TCCACCATGAACATGGAATC \\
TP63_e5 & GTTGGTTCTCTCCTTCCTTTC & GCCCACAGAATCTTGACCTTC \\
TP63_e6 & CCACCAACATCCTGTTCATGC & GTTCTCTCAAGTCTACTCAGTCC \\
TP63_e7 & GGGAAGAACTGAGAAGGAACAAC & CAGCCACGATTTCACTTTGCC \\
TP63_e8 & CAAGTGCTTTTGGGTCCATT & CACTTGCTGCTGAAGGTCAC \\
TP63_e9 & ATGCATTAGTGCTTTAGAAGT & GAAGGTTAAAATGAAGCAACC \\
TP63_e10 & TGAGGATTGACCACACTTCTAAC & CATCAATCACCCTATTGCTGATC \\
TP63_e11 & TGAACATCATTTCCATGTTTGTC & TCACAGAGTCTTGTCCTAAGC \\
TP63_e12 & GGACTATAACAGTATCCGCCC & CAAGATGGACCACTGGGATG \\
TP63_e13 & CTTATCTCGCCAATGCAGTTGG & AACTACAAGGCGGTTGTCATCAG \\
TP63_e14 & GGGAATGATAGGATGCTGTGG & AAGATTAAGCAGGAGTGCTT \\
DLX5_e1 & CTTAGACCAGAGCAGCCCC & ATCTACCACCCCATCTCGC \\
DLX5_e2 & TCTATTTGAGGCCCTTCCG & AGTCCCATCGAGACTGAACC \\
DLX5_e3 & GTCCGAAGATGCCTCCAGTC & CAGTTTTCCGAACTTCCCC \\
WNT10B_e2 & GTGTCTGATTGGGCAAGGTT & CTCATTGCTTAGAGCCCTGG \\
WNT10B_e3 & GGAGAGTTGGAGGGGTCTG & GAAACCATCCCTTCCCGC \\
WNT10B_e4 & TGCCTGTCAACCTTACCTCC & TAACCAGGCCTCAAAAGCTG \\
WNT10B_e5 & TGTGCCTCTGTGTTCTGTCC & GAAATCAGAGCAAAGGGCTG \\
\hline
\end{tabular}


whereas for proband's offspring is very low, unless there is consanguinity (Blattner et al. 2010). Noteworthy, autosomal recessive SHFM seems to be exceptionally rare. So far it has been reported only in a few, usually consanguineous families. Therefore, sequencing of WNT10 and DLX5 genes should be considered at the end of the diagnostic algorithm (for summary and diagnostic flow-chart proposed by us, see Fig. 3, for a list of primers routinely used to sequence $T P 63, W N T 10 B$ and $D L X 5$ genes, see Table 2).

Recent studies performed on a group of SHFLD cases and SHFM/SHFLD families have shown that duplications of 17 p13.3 with the minimal region of overlap encompassing only the single BHLHA9 gene are a frequent cause of isolated SHFM without deficiency of long bones (Klopocki et al. 2012). Importantly, from the clinical point of view, the penetrance in this locus is reduced to about $50 \%$ and the abnormality is often inherited from an unaffected parent. Of note, a strong sex bias has been observed with about $72 \%$ of the affected individuals being males. On the other hand females are usually asymptomatic carriers, but once they are affected they usually present with a more severe phenotype. The authors estimated the risk for a healthy $17 \mathrm{p} 13.3$ duplication carrier to have an affected male or female offspring as $36 \%$ and $15 \%$, respectively (Klopocki et al. 2012).

Development and dissemination of the molecular diagnostic testing, including aCGH have allowed for substantial improvement of the diagnostic yield in various groups of patients, including SHFM or other congenital limb defects. Genetic and clinical heterogeneity of SHFM contributes to the exceptionally difficult and challenging genetic counseling, including the recurrence risk assessment. Therefore, identification of the genetic alteration responsible for SHFM in individual patients is of practical importance for the entire family. First, it helps the affected or at risk individuals to understand the nature of the genetic condition as well as the options in management and preventive or supportive measures that may be available. Secondly, it provides a solid foundation for the actual risk estimation allowing for a conscious family planning and for prenatal or pre-implantation diagnosis. Finally, by the exclusion of all known causative alterations, diagnostic testing creates an opportunity to qualify unresolved cases for research testing (i.e., whole exome or whole genome sequencing), thereby contributing to the discovery of novel causes of SHFM.

Acknowledgements This work was supported by a grant from the National Science Centre (UMO-2011-03-D-NZ2-06136) to AJ. AS-S was supported by a grant from the Poznan University of Medical Science (502-14-01126186-08779), a scholarship support for Ph.D. students from the National Science Centre (UMO-2013/08/T/NZ2/00027) and a scholarship within the project: "Scholarship support for Ph.D. students specializing in major strategic development for Wielkopolska", Sub-measure 8.2.2 Human Capital Operational Programme, co-financed by the European Union under the European Social Fund.
Open Access This article is distributed under the terms of the Creative Commons Attribution License which permits any use, distribution, and reproduction in any medium, provided the original author(s) and the source are credited.

\section{References}

Acampora D, Merlo GR, Paleari L, Zerega B, Postiglione MP, Mantero S, Bober E, Barbieri O, Simeone A, Levi G (1999) Craniofacial, vestibular and bone defects in mice lacking the Distal-less-related gene Dlx5. Development 126(17):3795-3809

Ahmad M, Abbas H, Haque S, Flatz G (1987) X-chromosomally inherited split-hand/split-foot anomaly in a Pakistani kindred. Hum Genet 75(2):169-173

Aten E, den Hollander N, Ruivenkamp C, Knijnenburg J, van Bokhoven $\mathrm{H}$, den Dunnen J (2008) Split hand-foot malformation, tetralogy of Fallot, mental retardation and a $1 \mathrm{Mb} 19 \mathrm{p}$ deletion - evidence for further heterogeneity? Am J Med Genet A 152A(8):2053-2056

Bens S, Haake A, Tönnies H, Vater I, Stephani U, Holterhus PM, Siebert $\mathrm{R}$, Caliebe A (2011) A de novo $1.1 \mathrm{Mb}$ microdeletion of chromosome 19p13.11 provides indirect evidence for EPS15L1 to be a strong candidate for split hand split foot malformation. Eur J Med Genet 54(5):e501-e504

Birnbaum RY, Clowney EJ, Agamy O, Kim MJ, Zhao J, Yamanaka T, Pappalardo Z, Clarke SL, Wenger AM, Nguyen L, Gurrieri F, Everman DB, Schwartz CE, Birk OS, Bejerano G, Lomvardas S, Ahituv N (2012) Coding exons function as tissue-specific enhancers of nearby genes. Genome Res 22:1059-1068

Blattner A, Huber AR, Röthlisberger B (2010) Homozygous nonsense mutation in WNT10B and sporadic split-hand/foot malformation (SHFM) with autosomal recessive inheritance. Am J Med Genet A 152A(8):2053-2056

Boles RG, Pober BR, Gibson LH, Willis CR, McGrath J, Roberts DJ, Yang-Feng TL (1995) Deletion of chromosome 2q24-q31 causes characteristic digital anomalies: case report and review. Am J Med Genet 55(2): 155-160

Crackower MA, Scherer SW, Rommens JM, Hui CC, Poorkaj P, Soder S, Cobben JM, Hudgins L, Evans JP, Tsui LC (1996) Characterization of the split hand/split foot malformation locus SHFM1 at 7q21.3q22.1 and analysis of a candidate gene for its expression during limb development. Hum Mol Genet 5(5):571-579

Crackower MA, Motoyama J, Tsui LC (1998) Defect in the maintenance of the apical ectodermal ridge in the Dactylaplasia mouse. Dev Biol 201(1):78-89

Czeizel AE, Vitéz M, Kodaj I, Lenz W (1993) An epidemiological study of isolated split hand/foot in Hungary, 1975-1984. J Med Genet 30(7):593-596

de Mollerat XJ, Gurrieri F, Morgan CT, Sangiorgi E, Everman DB, Gaspari P, Amiel J, Bamshad MJ, Lyle R, Blouin JL, Allanson JE, Le Marec B, Wilson M, Braverman NE, Radhakrishna U, DelozierBlanchet C, Abbott A, Elghouzzi V, Antonarakis S, Stevenson RE, Munnich A, Neri G, Schwartz CE (2003a) A genomic rearrangement resulting in a tandem duplication is associated with split handsplit foot malformation 3 (SHFM3) at 10q24. Hum Mol Genet 12(16):1959-1971

de Mollerat XJ, Everman DB, Morgan CT, Clarkson KB, Rogers RC, Colby RS, Aylsworth AS, Graham JM Jr, Stevenson RE, Schwartz CE (2003b) P63 mutations are not a major cause of non-syndromic split hand/foot malformation. J Med Genet 40(1):55-61

Del Campo M, Jones MC, Veraksa AN, Curry CJ, Jones KL, Mascarello JT, Ali-Kahn-Catts Z, Drumheller T, McGinnis W (1999) Monodactylous limbs and abnormal genitalia are associated with 
hemizygosity for the human $2 \mathrm{q} 31$ region that includes the HOXD cluster. Am J Hum Genet 65(1):104-110

Dimitrov BI, de Ravel T, Van Driessche J, de Die-Smulders C, Toutain A, Vermeesch JR, Fryns JP, Devriendt K, Debeer P (2010) Distal limb deficiencies, micrognathia syndrome, and syndromic forms of split hand foot malformation (SHFM) are caused by chromosome 10q genomic rearrangements. J Med Genet 47(2):103-111

Duijf PH, van Bokhoven H, Brunner HG (2003) Pathogenesis of split-hand/split-foot malformation. Hum Mol Genet 12(Spec No 1):R51-R60

Elliott AM, Reed MH, Roscioli T, Evans JA (2005) Discrepancies in upper and lower limb patterning in split hand foot malformation. Clin Genet 68(5):408-423

Elliott AM, Evans JA (2006) Genotype-phenotype correlations in mapped split hand foot malformation (SHFM) patients. Am J Med Genet A 140(13):1419-1427

Everman DB, Morgan CT, Lyle R, Laughridge ME, Bamshad MJ, Clarkson KB, Colby R, Gurrieri F, Innes AM, Roberson J, Schrander-Stumpel C, van Bokhoven H, Antonarakis SE, Schwartz CE (2006) Frequency of genomic rearrangements involving the SHFM3 locus at chromosome 10q24 in syndromic and non-syndromic split-hand/foot malformation. Am J Med Genet A 140(13):1375-1383

Faiyaz ul Haque M, Uhlhaas S, Knapp M, Schüler H, Friedl W, Ahmad M, Propping P (1993) Mapping of the gene for X-chromosomal split-hand/split-foot anomaly to Xq26-q26.1. Hum Genet 91(1):17-19

Goodman FR, Majewski F, Collins AL, Scambler PJ (2002) A 117-kb microdeletion removing HOXD9-HOXD13 and EVX2 causes synpolydactyly. Am J Hum Genet 70(2):547-555

Gurrieri F, Kjaer KW, Sangiorgi E, Neri G (2002) Limb anomalies: Developmental and evolutionary aspects. Am J Med Genet 115(4): 231-244

Khan S, Basit S, Zimri FK, Ali N, Ali G, Ansar M, Ahmad W (2012) A novel homozygous missense mutation in WNT10B in familial splithand/foot malformation. Clin Genet 82(1):48-55

Ianakiev P, Kilpatrick MW, Toudjarska I, Basel D, Beighton P, Tsipouras P (2000) Split-hand/split-foot malformation is caused by mutations in the p63 gene on 3q27. Am J Hum Genet 67(1):59-66

Jamsheer A (2008) Genetic background of isolated forms of congenital malformations of the hand. Med Wieku Rozwoj 12(3):729-737

Jarvik GP, Patton MA, Homfray T, Evans JP (1994) Non-Mendelian transmission in a human developmental disorder: split hand/split foot. Am J Hum Genet 55(4):710-713

Johnson KR, Lane PW, Ward-Bailey P, Davisson MT (1995) Mapping the mouse dactylaplasia mutation, Dac, and a gene that controls its expression, mdac. Genomics 29(2):457-464

Klopocki E, Lohan S, Doelken SC, Stricker S, Ockeloen CW, Thiele S, de Aguiar R, Lezirovitz K, Mingroni Netto RC, Jamsheer A, Shah H, Kurth I, Habenicht R, Warman M, Devriendt K, Kordass U, Hempel M, Rajab A, Mäkitie O, Naveed M, Radhakrishna U, Antonarakis SE, Horn D, Mundlos S (2012) Duplications of BHLHA9 are associated with ectrodactyly and tibia hemimelia inherited in nonMendelian fashion. J Med Genet 49(2):119-125

Koster MI, Kim S, Mills AA, DeMayo FJ, Roop DR (2004) p63 is the molecular switch for initiation of an epithelial stratification program. Genes Dev 18(2):126-131

Kouwenhoven EN, van Heeringen SJ, Tena JJ, Oti M, Dutilh BE, Alonso ME, de la Calle-Mustienes E, Smeenk L, Rinne T, Parsaulian L, Bolat E, Jurgelenaite R, Huynen MA, Hoischen A, Veltman JA, Brunner HG, Roscioli T, Oates E, Wilson M, Manzanares M, Gómez-Skarmeta JL, Stunnenberg HG, Lohrum M, van Bokhoven H, Zhou H (2010) Genome-wide profiling of p63 DNA-binding sites identifies an element that regulates gene expression during limb development in the 7q21 SHFM1 locus. PLoS Genet 6(8):e1001065
Lewandoski M, Sun X, Martin GR (2000) Fgf8 signalling from the AER is essential for normal limb development. Nat Genet 26(4):460-463

Lezirovitz K, Maestrelli SR, Cotrim NH, Otto PA, Pearson PL, MingroniNetto RC (2008) A novel locus for split-hand/foot malformation associated with tibial hemimelia (SHFLD syndrome) maps to chromosome region 17p13.1-17p13.3. Hum Genet 123(6):625-631

Lo Iacono N, Mantero S, Chiarelli A, Garcia E, Mills AA, Morasso MI, Costanzo A, Levi G, Guerrini L, Merlo GR (2008) Regulation of Dlx5 and Dlx6 gene expression by p63 is involved in EEC and SHFM congenital limb defects. Development 135(7):1377-1388

Maniatis T (1999) A ubiquitin ligase complex essential for the NFkappaB, Wnt/Wingless, and Hedgehog signaling pathways. Genes Dev 13(5):505-510

Merlo GR, Paleari L, Mantero S, Genova F, Beverdam A, Palmisano GL, Barbieri O, Levi G (2002) Mouse model of split hand/foot malformation type I. Genesis 33(2):97-101

Mills AA, Zheng B, Wang XJ, Vogel H, Roop DR, Bradley A (1999) p63 is a 53 homologue required for limb and epidermal morphogenesis. Nature 398(6729):708-713

Moon AM, Capecchi MR (2000) Fgf8 is required for outgrowth and patterning of the limbs. Nat Genet 26(4):455-459

Qiu M, Bulfone A, Ghattas I, Meneses JJ, Christensen L, Sharpe PT, Presley R, Pedersen RA, Rubenstein JL (1997) Role of the Dlx homeobox genes in proximodistal patterning of the branchial arches: mutations of Dlx-1, Dlx-2, and Dlx-1 and -2 alter morphogenesis of proximal skeletal and soft tissue structures derived from the first and second arches. Dev Biol 185(2):165-184

Ramer JC, Mowrey PN, Robins DB, Ligato S, Towfighi J, Ladda RL (1990) Five children with del (2)(q31q33) and one individual with dup (2)(q31q33) from a single family: review of brain, cardiac, and limb malformations. Am J Med Genet 37:392-400

Robledo RF, Rajan L, Li X, Lufkin T (2002) The Dlx5 and Dlx6 homeobox genes are essential for craniofacial, axial, and appendicular skeletal development. Genes Dev 16(9):1089-1101

Seto ML, Nunes ME, MacArthur CA, Cunningham ML (1997) Pathogenesis of ectrodactyly in the Dactylaplasia mouse: aberrant cell death of the apical ectodermal ridge. Teratology 56(4):262-270

Shamseldin HE, Faden MA, Alashram W, Alkuraya FS (2012) Identification of a novel DLX5 mutation in a family with autosomal recessive split hand and foot malformation. $\mathrm{J}$ Med Genet 49(1):16-20

Sidow A, Bulotsky MS, Kerrebrock AW, Birren BW, Altshuler D, Jaenisch R, Johnson KR, Lander ES (1999) A novel member of the F-box/WD40 gene family, encoding dactylin, is disrupted in the mouse dactylaplasia mutant. Nat Genet 23(1):104-107

Simeone A, Acampora D, Pannese M, D'Esposito M, Stornaiuolo A, Gulisano M, Mallamaci A, Kastury K, Druck T, Huebner K, Boncinelli E (1994) Cloning and characterization of two members of the vertebrate $D l x$ gene family. Proc Natl Acad Sci U S A 91: 2250-2254

Sulik KK, Dehart DB (1988) Retinoic-acid-induced limb malformations resulting from apical ectodermal ridge cell death. Teratology 37(6): $527-537$

Tackels-Horne D, Toburen A, Sangiorgi E, Gurrieri F, de Mollerat X, Fischetto R, Causio F, Clarkson K, Stevenson RE, Schwartz CE (2001) Split hand/split foot malformation with hearing loss: first report of families linked to the SHFM1 locus in 7q21. Clin Genet 59(1):28-36

Temtamy SA, McKusick VA (1978) The genetics of hand malformations. Birth Defects Orig Artic Ser 14(3):i-xviii, 1-619

Ugur SA, Tolun A (2008) Homozygous WNT10b mutation and complex inheritance in Split-Hand/Foot Malformation. Hum Mol Genet 17(17):2644-2653

van Bokhoven H, Hamel BC, Bamshad M, Sangiorgi E, Gurrieri F, Duijf PH, Vanmolkot KR, van Beusekom E, van Beersum 
SE, Celli J, Merkx GF, Tenconi R, Fryns JP, Verloes A, Newbury-Ecob RA, Raas-Rotschild A, Majewski F, Beemer FA, Janecke A, Chitayat D, Crisponi G, Kayserili H, Yates JR, Neri G, Brunner HG (2001) p63 Gene mutations in eec syndrome, limb-mammary syndrome, and isolated split hand-split foot malformation suggest a genotype-phenotype correlation. Am J Hum Genet 69(3):481-492

van Silfhout AT, van den Akker PC, Dijkhuizen T, Verheij JB, OlderodeBerends MJ, Kok K, Sikkema-Raddatz B, van RavenswaaijArts CM (2009) Split hand/foot malformation due to chromosome $7 \mathrm{q}$ aberrations (SHFM1): additional support for functional haploinsufficiency as the causative mechanism. Eur J Hum Genet 17(11):1432-1438

Vaudin P, Delanoue R, Davidson I, Silber J, Zider A (1999) TONDU

(TDU), a novel human protein related to the product of vestigial (vg) gene of Drosophila melanogaster interacts with vertebrate TEF factors and substitutes for $\mathrm{Vg}$ function in wing formation. Development 126(21):4807-4816

Wong WT, Schumacher C, Salcini AE, Romano A, Castagnino P, Pelicci PG, Di Fiore PP (1995) A protein-binding domain, $\mathrm{EH}$, identified in the receptor tyrosine kinase substrate Eps 15 and conserved in evolution. Proc Natl Acad Sci U S A 92(21):9530-9534

Yang Y (2003) Wnts and wing: Wnt signaling in vertebrate limb development and musculoskeletal morphogenesis. Birth Defects Res C Embryo Today 69(4):305-317

Yang A, Schweitzer R, Sun D, Kaghad M, Walker N, Bronson RT, Tabin C, Sharpe A, Caput D, Crum C, McKeon F (1999) p63 is essential for regenerative proliferation in limb, craniofacial and epithelial development. Nature 398(6729):714-718 